\title{
Collisional and Chaotic Transport of Energetic Particles in Toroidal Plasma
}

John R. Cary, Principal Investigator

Svetlana G. Shasharina, Co-Investigator

DOE/ER/53207--7

DE92 014589

Department of Astrophysical, Planetary, and Atmospheric Sciences

University of Colorado

Boulder, CO 80309-0391

April, 1992

\begin{abstract}
We have made progress in three general areas of confinement plasma physics is proposed. (1) We developed an accurate theory for the trapping/detrapping process in tokamaks with ripple and stellarators. (2) We determined the alpha particle distribution in the situation where both collisions and collisionless chaotic (ripple) transport are present.
\end{abstract}

\section{DISCLAIMER}

This report was prepared as an account of work sponsored by an agency of the United States Government. Neither the United States Government nor any agency thereof, nor any of their employees, makes any warranty, express or implied, or assumes any legal hability or respons, or bility for the accuracy, completeness, or usefulness of any information, apparatus, product, process disclosed, or represents that its use would not infringe prive by trade name, trademark, ence herein to any specific commercial product, process, or service by trade its endorsement, recommanufacturer, or otherwise does not necessarily constitute or any agency thereof. The views mendation, or favoring by the United States Government or any state or reflect those of the and opinions of authors expressed herein do not

$F G 6.2-8.5 E R 532070$ 


\section{Table of Contents}

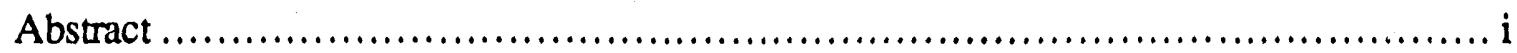

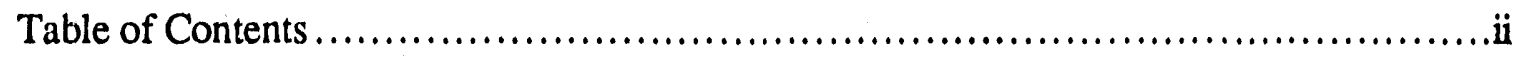

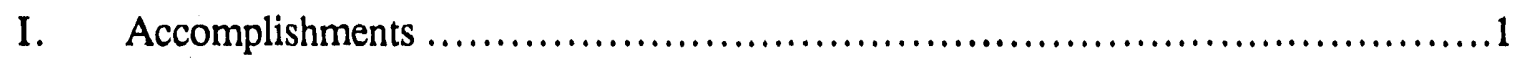

II. Trapping probability in asymmetric tori ........................................

III. Stochastic loss of alphas in tokamak............................................6

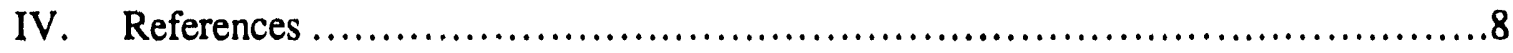

Appendix A. Probability of Orbit Transition in Asymmetric Toroidal Plasma

Appendix B. Stochastic Losses of Alphas in Tokamaks 
Research in three general areas of confinement plasma physics was performed. The main effort was to reexamine the collisionless motion of the particles in asymmetric toroidal devices to be able to formulate the boundary conditions for the distribution function at low collision frequency. Then, we investigated the transport of suprathermal particles (i.e. alpha particles) in the regime where the effects of both collisions and collisionless chaotic transport were taken into account. In addition, we continued our developing symplectic integration methods.

Studies of transport in asymmetric toroidal devices, e.g. stellarators and tokamaks with ripple, in the low-collisionality regime have been a subject of intense attention in recent years. These investigations are necessary for the understanding of fusion reactors, which must operate at high temperature, where the collision frequency for the particles of the bulk plasma is small. These investigations are also important for smaller experimental devices with neutral beam injected particles.

To carry out the study of the low-collisional transport, we first need to investigate the collisionless motion of the particles in the magnetic fields of the rippled tokamak and stellarator. This motion is not trivial because of the existence of the transitioning particles. ${ }^{1-4}$ These particles make transitions between the locally trapped state and the toroidally trapped state even without collisions. Since these particles can have large radial displacements from the magnetic surfaces, these particles contribute significantly to transport. Hence, an accurate transport theory, either analytical or numerical, must include the evaluation of the trapping probability, the fraction of a toroidally trapped phase uniform distribution that makes a transition to the trapped state at the end of the bounce motion.

In Sec. II, we present a new calculation of the transition probability. The original motivation for re-examining the transition probability calculations was our numerical work 
which showed that previous calculations $s^{1-4}$ of the transition probability are inaccurate for realistic parameters. Our new method differs from previous by using a more accurate model of the field variation near the transition and by including in lowest order the poloidal motion due to finite rotational transform.

Another area of our interest is related to alpha particles. The confinement of these alphas is critical to the development of fusion reactors. Our goal here is to include both collisional and collisionless mechanisms of the losses into Fokker-Planck equation. The collisional mechanism is slowing down due to friction on the background electrons and change of the pithch angle due to scattering off the background ions. ${ }^{5,6}$ The collisionless loss occurs on a time scale much shorter then any of the collisional times and is related to the dynamic stochasticity ${ }^{7,8}$ of the orbits arising due to magnetic ripple. That is why the stochastic losses are accounted for by assuming that all the stochastic particles are lost immediately. We present the solution of the Fokker Plank equation for the supra thermal particles in Sec.3. 
II. Trapping probability in asymmetric tori

To investigate the motion of the particles in magnetic confinement devices, tokamak and stellarator, we use the standard model, in which the magnetic field strength has the form,

$$
\left.B=B_{0}\left[1-\varepsilon_{t}(\psi) \cos (\theta)-\varepsilon_{r}(\psi) \cos (N \phi-l \theta)\right)\right]
$$

The numbers $l$ and $\mathrm{N}$ are the poloidal and toroidal mode numbers for a stellarator, while for a tokamak $l=0 ; \psi, \theta, \phi$ are traditional Boozer coordinates. ${ }^{2}$

There are two types of trapped particle orbits, if the drift to neighboring field lines is neglected: the toroidally trapped and the locally trapped particles. The inclusion of drifts allows the toroidally trapped particles to become locally trapped. The slow change of $\psi$ and $\theta$ causes the ripple amplitude and toroidicity to change, while the slow change of $\theta$ causes the phase between the fast and slow components of the magnetic field to change. Due to these effects, a toroidally trapped particle, after passing over the last local maximum near its bounce point, may be trapped by that last maximum. Conversely, a locally trapped particle may result in the decrease of the local maximum, thus allowing the particle to escape the local magnetic well.

We define the trapping probability, $\mathrm{P}$, as a ratio of the flux of the particles trapped into the magnetic well to the total flux reflecting between the points, $\phi_{0}$ and $\phi_{1}$, which are the coordinares of the maxima of the well. We show that $\mathrm{P}$ can be represented as

$$
P_{r}=\left[E_{0}, Y_{0}\right] /\left(\Delta E_{x}(1-l t / N)+\frac{1}{2}\left[E_{1}, Y_{1}\right]\right\}
$$

where

$$
Y_{0} \equiv \oint_{E=E_{0}} v_{\varphi} d \varphi
$$




$$
\begin{aligned}
& Y_{1}=2 \int_{\varphi_{0}}^{\varphi_{1}} d \varphi v_{\varphi}, \\
& \Delta E_{x}=E_{1}-E_{0} .
\end{aligned}
$$

Here, $E_{0}$ and $E_{1}$ are the values of energy on the local maxima $\phi_{0}$ and $\phi_{1}$, and $v_{\varphi}=u B_{0} / B$ with $u$ being the parallel velocity. The Poisson bracket used in Eq.(3) is defined as

$$
[a, b]=\frac{\partial a}{\partial \psi} \frac{\partial b}{\partial \theta} \cdot \frac{\partial a}{\partial \theta} \frac{\partial b}{\partial \psi} \text {. }
$$

The main novelties in Eq.(2) in comparison with most of the prevous papers are: (1) we include the additional flux of the particles arising due to drift which also contributes to the total incoming flux (the denominator), (2) we include the correct Lamé coefficient, $\mathrm{B}_{0} / \mathrm{B}$, into the element of the length, and (3) we distinguish between the values $E_{0}$ and $E_{1}$ which are different due to the finite rotational transform. The rotational transform implies that, on the magnetic field line and, hence, in Eq.(2), the poloidal and toroidal angles are related by

$$
\theta=\theta_{0}+t \phi
$$

To calculate the integrals in Eqs.(3) and (4) we model the magnetic field, Eq.(1), along the field line by

$$
\mathrm{B} / \mathrm{B}_{0}=\left\{\begin{array}{ll}
1-\varepsilon_{\mathrm{t}} \cos \left(\theta_{0}\right)-\Delta_{0} \cos \left(\mathrm{k}_{0}\left(\phi-\phi_{\mathrm{mn}}\right)\right) & \text { for } \phi_{0}<\phi<\phi_{\mathrm{m} n} \\
1-\varepsilon_{\mathrm{t}} \cos \left(\theta_{0}\right)-\Delta_{1} \cos \left(\mathrm{k}_{1}\left(\phi-\phi_{\mathrm{mn}}\right)\right) & \text { for } \phi_{\mathrm{m} n}<\phi<\phi_{1}
\end{array}\right. \text {, }
$$

where $\Delta_{0}\left(\Delta_{1}\right)$ is the height of the left (right) maximum of the magnetic field relative to the minimum achieved at $\phi_{m n}$, and $k_{i}$ is the contraction factor, $k_{i}=\pi /\left(\phi_{i}-\phi_{m n}\right)$. Applying this model to Eqs. (2)-(4), we obtain analytic expressions for $Y_{0}, Y_{1}$ and the trapping probability, $\mathrm{P}$, in terms of elliptic integrals. 
We compare the trapping probability obtained by use of the adiabatic theory with direct numerical integration of the guiding center equations. The trajectories are started at $\theta=0$ for various values of $\varphi$ with fixed initial value of the energy and the first-ordercorrected adiabatic invariant. We determine the range of initial values of $\varphi$ for which trajectories reflect or become trapped. Ultimately we find two initial values, $\varphi_{\mathrm{T} 1}$ and $\varphi_{\mathrm{T} 2}^{\prime}$, such that trajectories launched between $\varphi_{\mathrm{T} 1}$ and $\varphi_{\mathrm{T} 2}$ (modulo $2 \pi / \mathrm{N}$ ) become trapped, while trajectories launched between $\varphi_{\mathrm{T} 2}$ and $\varphi_{\mathrm{T} 1}$ (modulo $2 \pi / \mathrm{N}$ ) reflect. The numerical trapping probability is then evaluated as a ratio of the fluxes limited by $\varphi_{\mathrm{T} 1}$ and $\varphi_{\mathrm{T} 2}$ to the flux in full period.

For the case depicted on Fig. 9 of App. A, we use parameters which are ATF-like: $\delta_{\mathrm{r}}=0.21, \delta_{\mathrm{t}}=0.14, \varepsilon_{\mathrm{r}}=\delta_{\mathrm{r}} \rho^{2}$ and $\varepsilon_{\mathrm{t}}=\delta_{\mathrm{t}} \rho$, where $\rho$ is the minor radius, $t=0.35+0.55 \rho^{2}$. The electrostatic potential is assumed to have parabolic form: $\Phi=\Phi_{0} \rho^{2}$. The energy parameters, $\gamma_{\mu} \equiv \mu \mathrm{B}_{0} / \mathrm{m}(\Omega \mathrm{a})^{2}, \quad \gamma_{\mathrm{E}} \equiv \mathrm{E} / \mathrm{m}(\Omega \mathrm{a})^{2}$, and $\gamma_{\Phi} \equiv \mathrm{q} \Phi_{0} / \mathrm{m}(\Omega \mathrm{a})^{2}$, are $\gamma_{\mu}=1.81 \times 10^{-4}, \gamma_{E}=3 \times 10^{-4}$, and $\gamma_{\Phi}=2 \times 10^{-4}$ with $N=12$.

Our numerical analysis shows that the new approach to evaluate the trapping probability, as well as the model of the magnetic field,the adiabatic invariant are superior to those used in the previous papers because it overall give the best prediction for the trapping probability. 
III. Stochastic loss of alphas in tokamak

In the presence of asymmetric magnetic fields, such as ripple, the trajectories of fast particles can be chaotic. These chaos arises due to resonance between the toroidal precession and the bounce frequency. The stochastic diffusion rate exceeds other mechanisms by order of magnitude. Hence, the determination of the fast particle loss due to stochasticity is crucial for knowing whether alphas can be well confined. We reexamined the motion of alpha particles in rippled magnetic fields in order to determine the distribution and heat deposition of fusion produced alphas in the presence of both chaotic losses and collisional redistribution of pitch angle and energy.

To model the fast chaotic losses we impose the boundary condition that the particle distribution vanishes in the stochastic region of the velocity space. This region is specified by the Goldston-White-Boozer criterion,

$$
\xi<\xi_{\mathrm{t}}, \quad \mathrm{v}>\mathrm{v}_{\mathrm{S}},
$$

with

$$
\mathrm{v}_{\mathrm{S}}=\frac{\Omega}{2 \varepsilon_{\mathrm{r}}}\left(\frac{\varepsilon_{\mathrm{t}}}{\pi \mathrm{Nq}}\right)^{3 / 2} \frac{1}{\mathrm{dq} / \mathrm{dr}}
$$

where $\mathrm{q}=1 / t$ and $\xi=\left[1-2 \mu \mathrm{B}_{\mathrm{o}} / \mathrm{v}^{2}\right]^{1 / 2}$, and $\mathrm{r}$ is the minor radius of the torus. Eqs.(9) and (10) mean that the particle should be toroidally trapped and sufficiently fast. We impose these boundary conditions to the stationary drift kinetic equation,

$$
\frac{u \varepsilon_{t}}{q r}=C_{P}(f)+C_{v}(f)+S_{0} \delta\left(v \cdot v_{0}\right)
$$

with the Lorentz collision operator, $C_{P}(f)$, slowing down term, ${ }^{5,6} C_{v}(f)$, and the source, $S_{0} \delta\left(v-v_{0}\right)$. 
We assume that the radial width of the banana orbit is small. We apply Cordey's procedure of expanding in the ratio of the collision frequency to the bounce frequency. Then the lowest order term, $\mathrm{f}_{0}$, can be found by use of the solubility condition and separable expansion, $f_{0}=\sum_{n} a_{n}(v) C_{n}(\xi)$, in the eigen-functions of the operator $C_{P}(f) / u$ averaged along the trajectory. We then evaluate the relative flux of the particles into stochastic region gives the relative stochastic loss of alphas,

$$
\gamma_{S t}=-<\int_{M} C_{p}\left(f_{o}\right) d^{3} v>/\left(4 p v_{0}^{2} S_{0}\right)
$$

where $M$ is the the velocity space with $v<v_{0}$ and without stochastic loss cone, and the angular brackets denote the magnetic surface averaging.

For the "typical" case, i.e. ITER design, we found that the alpha particle loss constitutes approximately $60 \%$ of all the particles and the energy loss is up to $15 \%$. 
IV. References

1 R.J. Goldston and H.H Towner, J. Plasma Phys. 26, 283 (1981).

2 J.R. Cary, C.L. Hedrick, and J.S. Tolliver, Phys. Fluids. 31, 1586 (1988).

3 V.P. Nagornij and V.A. Yavorskij, Sov.J. Plasma Phys. 15, 309 (1989).

4 L.M. Kovrizhnykh and S.G. Shasharina, Nucl. Fusion 30, 453 (1990).

5 J.D. Cordey, Nucl. Fusion 16, 199 (1976).

6 C.T. Hsu, P.J. Catto, and D.J. Sigmar, Phys. Fluids B2, 280 (1990).

7 R. J. Goldston, R. B. White, and A. H. Boozir, Phys. Rev. Lett. 47, 647 (1981).

8 R. B. White and H. E. Mynick, Phys. Fluids B 1, 980 (1989).

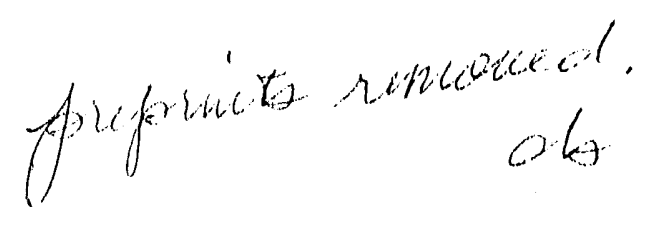




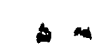
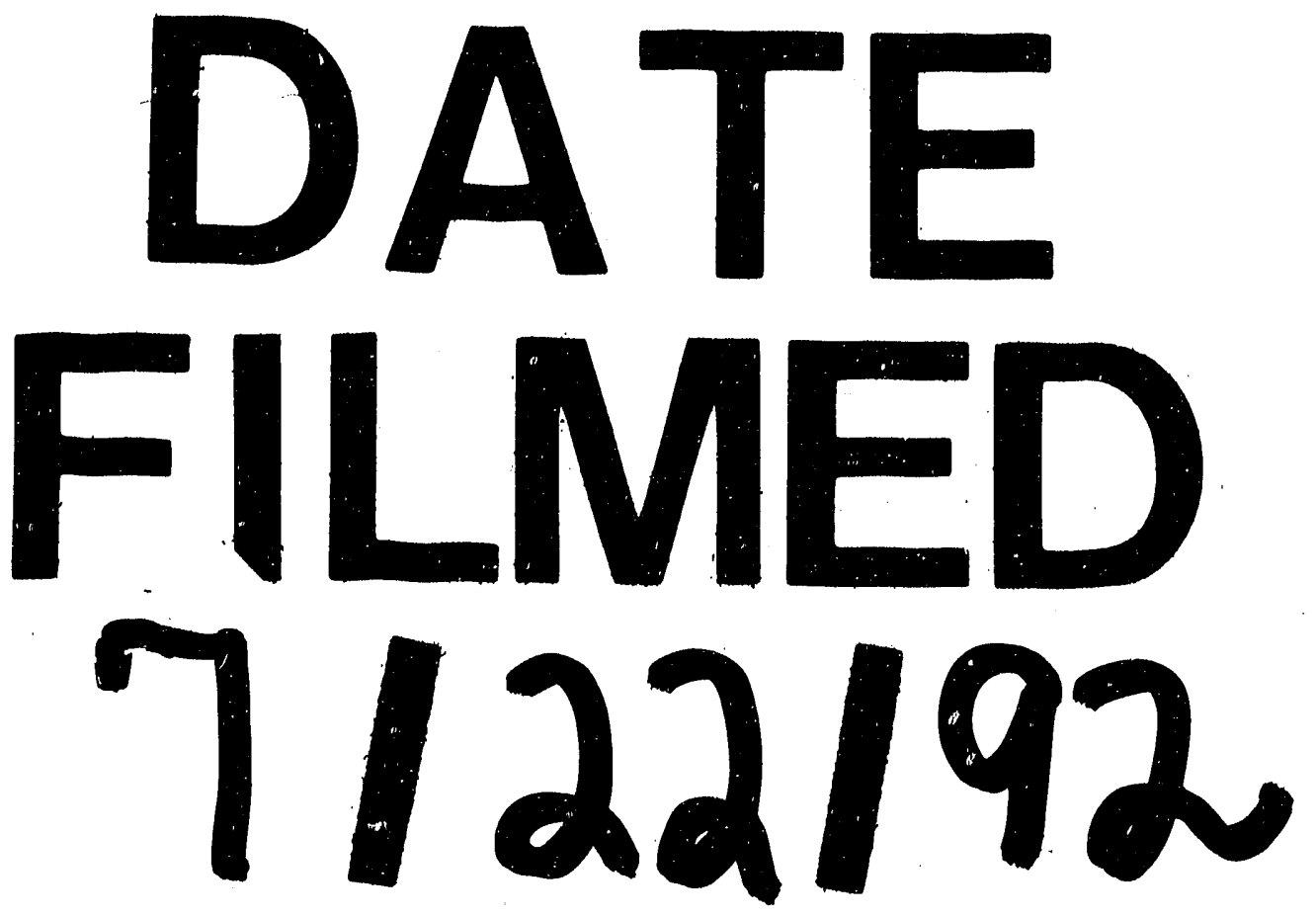

- : 4 
